\title{
Diabetes care for the elderly: still room for improvement
}

Physicians who care for the elderly are increasingly reminded that diabetes is not a mild condition in old age. It has been demonstrated that both macrovascular and microangiopathic complications are highly prevalent in elderly diabetics. 1,2 This in turn implies high levels of disability, although there are few studies directly measuring this. With its multisystem pathology, potential for great disability and increasing prevalence with advancing age, diabetes mellitus is a legitimate interest for the geriatrician. Many of us however may be unsure of what care to give our elderly diabetic patients or how best to deliver it. We hear of advances in determining the genetic predisposition to non-insulin dependent diabetes (NIDDM) and the relative contributions of insulin resistance and beta cell failure. On the other hand, we see debate about the optimum diabetic diet, doubts as to whether complications can be reduced and the view that diabetes diagnosed in the eighth decade of life carries no excess mortality (i.e. the 'diabetes is a mild disease in old age' view persists).

The findings of the Diabetes Control and Complications Trial Research Group (DCCT) ${ }^{3}$ of a reduction in diabetic complications in young insulin-dependent diabetics by optimizing glycaemic control has encouraged those who feel more effort should be made to reduce complications in NIDDM. Therapeutic nihilists will point out that conclusions drawn from a trial in young IDDM patients should not necessarily apply to the elderly. They will also remind us of the high incidence of hypoglycaemic episodes in DCCT which would be less acceptable to an elderly population. This latter worry has been an argument against optimizing glycaemic control in the elderly along with the belief that they are less able to perform self blood glucose monitoring as a safeguard. A recent small study 4 has, however, suggested that the elderly are just as able as young diabetics to learn and perform self blood glucose monitoring. In further support of the therapeutic optimists, a Japanese study of progression of mild retinopathy in elderly subjects with NIDDM (mean age $68 \pm 6$ years) published recently 5 showed that those with mean glycosylated haemoglobin levels nearest normal had the least progression of retinopathy over a five year period.

An optimistic geriatrician might conclude that, in co-operation with the general practitioner and diabetic specialist nurse, it is both reasonable and relatively safe to optimize glycaemic control to both relieve symptoms and possibly reduce future complications. He is however, often frustrated by the fact that NIDDM is asymptomatic in the early stages or that many elderly people attribute their symptoms to aging and the condition does not present early. We are all sadly familiar with this condition presenting acutely with one of its complications but would hope that improved health screening in primary care may ultimately detect diabetes earlier and reduce this type of disaster. The interested geriatrician is also less able to make an impression on complication rates because he inherits patients from colleagues in primary and secondary care who had the greatest 


\section{Editorial}

opportunities for patient education and complication prevention - the damage has been done!

If the 'physician in care of the elderly with an interest in diabetes' remains undeterred, how should they organize care for the elderly diabetic and who should they see? In setting up a separate clinic for older diabetics, the physician runs the risk of being overwhelmed by numbers and providing no more time for assessment and education than traditional diabetic clinics. Holding a clinic in the general outpatients department of many hospitals might put physical constraints on what can be achieved with elderly diabetics. Day hospitals are often a better venue in terms of layout, time available, philosophy of care of chronic disabling disease, and access to rehabilitation staff. Here the nursing staff also have experience of foot and ulcer care and chiropodists may already visit. Whilst places are becoming available in many day hospitals due to changes in care of other conditions, it may be less than straightforward to set up a diabetic clinic there. Patients attend on various days from different areas to facilitate ambulance schedules making it difficult to organize a consultant-based clinic for elderly diabetics in every day hospital every day.

As we cannot hope to see all elderly diabetics we might choose to concentrate our efforts on those most disabled. This is entirely appropriate as we are expert in assessing multiple problems, identifying disabilities and co-ordinating patient care with multidisciplinary teams. We should not feel satisfied with this alone if we recall that geriatricians have been shown to be less thorough than diabetologists in performing simple screening routines for complications, for example, visual acuity. Whilst we may well see patients with advanced diabetes in whom testing for proteinuria for example seems pointless, we are still in a position to prevent blindness by identifying proliferative retinopathy and making prompt referral. This of course assumes regular practice of fundoscopy through dilated pupils! We are also in a position to prevent foot trauma with appropriate inspection of feet and footwear coupled with adequate patient education.

No matter where a geriatrician sees a diabetic patient he might usefully view that patient as potentially very disabled and as such we have a lot to offer. Whilst it is debatable whether all diabetic complications are preventable in the patients geriatricians care for, it is clear that further disability can be avoided if we take care to perform adequate eye and foot examinations. Don't forget the tropicamide and look under the desk!

Colin Jenkins, Senior Registrar in Geriatric Medicine Age Care, General Hospital, Hereford HR1 2PA, UK.

\section{References}

1 Neil HAW, Thompson AV, Thoroughgood M, Fowler GH, Mann JL. Diabetes in the elderly: the Oxford community diabetes study. Diabetic Med 1989; 6: 608-13.

2 Dornan T, Peck GM, Dow JDC, Tatersall RB. A community survey of diabetes in the elderly. Diabetic Med 1992; 9: 860-65.

3 The Diabetes Control and Complications Trial Research Group. The effect of intensive treatment of diabetes on the development and progression of long-term complications in insulin-dependent diabetes mellitus. N Engl J Med 1993; 329: 977-86.

4 Bernbaum M, Albert SG, McGinnis J, Brusca S, Mooradian AD. The reliability of self blood glucose monitoring in elderly diabetic patients. J Am Geriatr Soc 1994; 42: 779-81.

5 Morisaki N, Watanabe S, Kobayashi J et al. Diabetic control and progression of retinopathy in elderly patients: five-year follow-up study. J Am Geriatr Soc 1994; 42: 142-45. 